\title{
Model-Reference Discretized PID Control and Robust Stabilization for Continuous Plants
}

\author{
Yoshifumi Okuyama* \\ * Humanitech Laboratory, Co., Ltd. \& Tottori University(Emeritus), Japan \\ (Tel: +81-88-625-2545; e-mail: oku@humanitech-lab.jp).
}

\begin{abstract}
This paper deals with a designing problem of discrete-time and discrete-value (discretized) control systems based on a model-reference feedback structure. The model used in this study is assumed to be a second-order lag system that is expressed by a bilinear transformation. That type of discretized (nonlinear) control systems is presented and analyzed. The model reference feedback using a secondorder continuous-value (linear) system is equivalently transformed into a traditional PID control. The robust stability analysis and design of such nonlinear control systems is examined in a frequency domain. Numerical examples for model-reference type discretized control are provided to verify the design method.
\end{abstract}

Keywords: Nonlinear systems; robust stability; discretization; quantization; model reference control.

\section{INTRODUCTION}

Almost all feedback control systems are realized using discretized (discrete-time and discrete-value) signals. However, the analysis and design of discretized/quantized control systems has not been entirely elucidated. An attempt to elucidate the quantized control systems was presented first in a paper (Kalman [1956]). Since then, the problem of mitigating the quantization effects in quantized control systems has been studied (Delchamps [1990], Elia and Mitter [2001], Fu [2003]). However, few results have been obtained for the analysis and design of that type of nonlinear discrete-time control systems (Desoer and Vidyasagar [1975]).

In our previous papers (Okuyama [2006, 2008, 2009]), the robust stabilization of nonlinear discrete-time and discrete-value (discretized) control systems was examined in a frequency domain. In the study, a traditional (discrete-time) PID control scheme was used in the controller design. In the design procedure, the concept of a modified Nyquist and Hall diagram (off-axis M-circles) for nonlinear control systems in (Okuyama et al. [2002a]) is applied.

This paper describes a designing problem of such discretized control systems based on a model-reference feedback structure. The model-reference feedback using a second-order continuous-value (linear) system is equivalently transformed into a traditional PID control. This control scheme is referred to as a quasi-PID control in this paper. The robust stability analysis and design of such nonlinear control systems is examined in a frequency domain.

\section{BILINEAR TRANSFORMATION AND MODEL REFERENCE FEEDBACK}

Model reference feedback structure for a robust control system was once proposed by the author. A discrete-time version of the model reference feedback was presented in (Takemori and Okuyama [2000]). In this paper, a discretized model reference control system as shown in Fig. 1 is examined. Here, $P(s)$ is a continuous-time and continuous-value plant, and $P(z)$ is the $z$-transform of $P(s)$ together with the zero-order hold $\mathcal{H}$. On

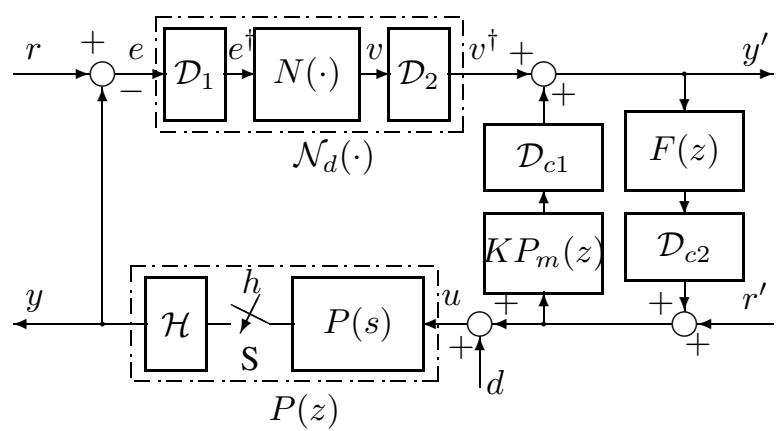

Fig. 1. Model-reference discrete control system.

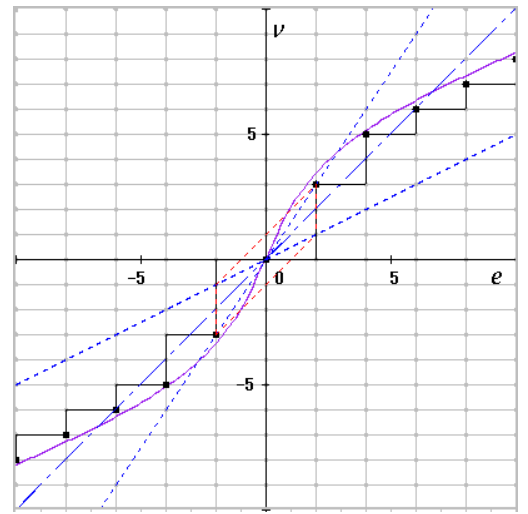

Fig. 2. Discretized nonlinear characteristics $(K=1.0)$.

the other hand, $N(\cdot), \mathcal{D}_{1}$ and $\mathcal{D}_{2}$ are a nonlinear element and the input and output discretizing units which are determined by sensor and actuator elements. In the figure, each symbol $e, v$, $\cdots$ indicates the sequence $e(k), v(k), \cdots,(k=0,1,2, \cdots)$ in discrete time, but for continuous value. On the other hand, each symbol $e^{\dagger}, v^{\dagger}, \cdots$ indicates a discrete value that can be assigned to an integer number, e.g.,

$$
\begin{aligned}
& e^{\dagger} \in\left\{\cdots,-2 \gamma_{1},-\gamma_{1}, 0, \gamma_{1}, 2 \gamma_{1}, \cdots\right\}, \\
& v^{\dagger} \in\left\{\cdots,-2 \gamma_{2},-\gamma_{2}, 0, \gamma_{2}, 2 \gamma_{2}, \cdots\right\},
\end{aligned}
$$


where $\gamma_{1}$ and $\gamma_{2}$ are the resolution of each variable. The discretized nonlinear characteristic $v^{\dagger}=\mathcal{N}_{d}(\cdot)$ is depicted as shown in Fig. 2. The resolutions in $\mathcal{D}_{1}$ and $\mathcal{D}_{2}$ are chosen as $\gamma_{1}=2.0$ and $\gamma_{2}=1.0$ in this example. $\mathcal{D}_{c 1}$ and $\mathcal{D}_{c 2}$ are the discretizing units in the discrete-time model $K P_{m}(z)$ and the feedback compensator $F(z)$. Those resolutions are considered $\gamma_{c 1}=\gamma_{c 2}=\gamma_{2} / n$. Thus, when $n \rightarrow \infty$, the controller part can be considered a linear continuous system.

In this study, the model system, $K P_{m}(z)$ ( $K$ : nominal linear gain of $\left.\mathcal{N}_{d}(\cdot)\right)$, is assumed to be a second-order lag system, e.g.,

$$
K P_{m}(z)=K \tilde{P}_{m}(\delta)=\frac{K}{1+C_{1} \delta+C_{2} \delta^{2}},
$$

where $\delta$ is the following bilinear transformation:

$$
\delta=\frac{2}{h} \cdot \frac{z-1}{z+1} \quad(h: \text { sampling period }) .
$$

Here, $C_{1}$ and $C_{2}$ are the design parameters of the model system.

The $z$-transform expression of (2) is given by

$K P_{m}(z)=\frac{K h^{2}(z+1)^{2}}{h^{2}(z+1)^{2}+2 C_{1} h(z+1)(z-1)+4 C_{2}(z-1)^{2}}$.

Obviously, $\delta$ approaches Laplace transform variable $s$, when the sampling period is $h \rightarrow 0$.

The operator $\delta$ has the following properties:

(1) Since $\delta^{-1}=\frac{h}{2} \cdot \frac{1+z^{-1}}{1-z^{-1}}$, the relationship between the input and output sequences, $x(k), y(k),(k=0,1,2, \cdots)$ can be written as:

$$
y(k)=y(k-1)+\frac{h}{2}(x(k)+x(k-1)) .
$$

This transformation corresponds to a trapezoidal summation (integration).

(2) On the other hand, with respect to the operator $\delta$, the following relation can be obtained:

$$
y(k)=-y(k-1)+\frac{2}{h}(x(k)-x(k-1)) .
$$

In this paper, $\delta$ will be used instead of $z$-transform operator. Therefore, the feedback compensator $F(z)$ as shown in Fig. 1 is defined as:

$$
F(z)=\tilde{F}(\delta)=\frac{1+C_{1} \delta+C_{2} \delta^{2}}{K\left(1+c_{1} \delta+c_{2} \delta^{2}\right)} .
$$

Here, $c_{1}$ and $c_{2}$ are considered the design parameters of the feedback compensator. Thus, the $z$-transform expression of (7) is written as:

$F(z)=\frac{h^{2}(z+1)^{2}+2 C_{1} h(z+1)(z-1)+4 C_{2}(z-1)^{2}}{K\left(h^{2}(z+1)^{2}+2 c_{1} h(z+1)(z-1)+4 c_{2}(z-1)^{2}\right)}$.

In the frequency domain, $\delta$ can be expressed as:

$$
\delta\left(\mathrm{e}^{j \omega h}\right)=j \Omega(\omega)=j \frac{2}{h} \tan \left(\frac{\omega h}{2}\right), \quad j=\sqrt{-1},
$$

where $\Omega$ is a distorted frequency of $\omega$. Using this expression, (2) and (7) can be written as follows:

and

$$
K P_{m}\left(\mathrm{e}^{j \omega h}\right)=K \tilde{P}_{m}(j \Omega)=\frac{K}{1-C_{2} \Omega^{2}+j C_{1} \Omega},
$$

$$
F\left(\mathrm{e}^{j \omega h}\right)=\tilde{F}(j \Omega)=\frac{1-C_{2} \Omega^{2}+j C_{1} \Omega}{K\left(1-c_{2} \Omega^{2}+j c_{1} \Omega\right)} .
$$

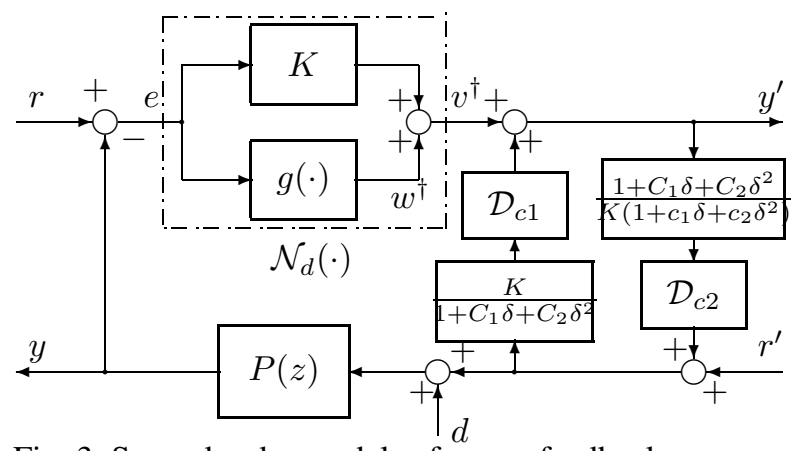

Fig. 3. Second order model-reference feedback.

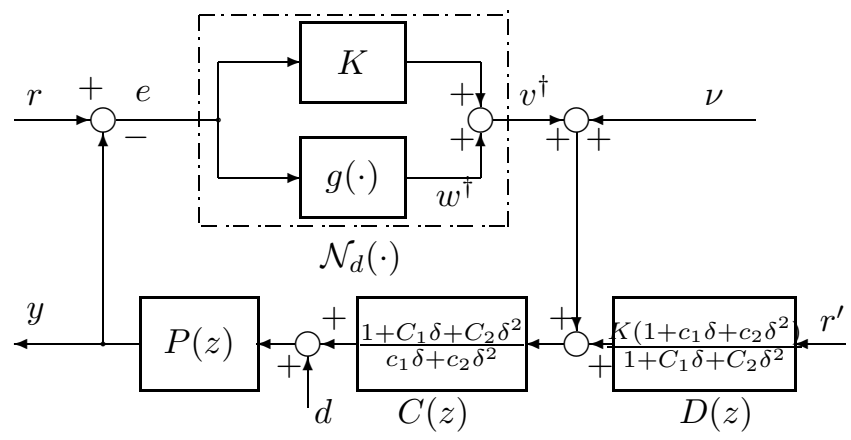

Fig. 4. Quasi-PID control system.

The block diagram of model-reference control systems can be redrawn as shown in Fig. 3.

\section{QUASI-PID CONTROL AND ITS IMPROVEMENT}

When controllers are in high resolution, i.e., $n \rightarrow \infty$, the model-reference control system as shown in Fig. 1 can be transformed into Fig. 4. Here, $\nu$ is a disturbance signal generated by the discretization of controllers. The equivalent controller $C(z)$ and the pre-compensator $D(z)$ are given by

$$
\begin{gathered}
C(z)=\tilde{C}(\delta)=\frac{1+C_{1} \delta+C_{2} \delta^{2}}{c_{1} \delta+c_{2} \delta^{2}}, \\
D(z)=\tilde{D}(\delta)=\frac{K\left(1+c_{1} \delta+c_{2} \delta^{2}\right)}{1+C_{1} \delta+C_{2} \delta^{2}} .
\end{gathered}
$$

Here, $C(z)$ can be considered to be a controller when $c_{2} \ll c_{1}$. If $c_{2} \rightarrow 0$, the controller is approximately written as:

$$
\tilde{C}(\delta)=\frac{1}{c_{1}} \delta^{-1}+\frac{C_{1}}{c_{1}}+\frac{C_{2}}{c_{1}} \delta .
$$

When the sampling period is $h \rightarrow 0, \delta$ becomes Laplace transform variable $s$. Therefore, the scheme will correspond to a traditional continuous PID control.

The $z$-tranform of (14) is given by

$$
C(z)=\frac{h}{2 c_{1}} \cdot \frac{z+1}{z-1}+\frac{C_{1}}{c_{1}}+\frac{2 C_{2}}{c_{1} h} \cdot \frac{z-1}{z+1} .
$$

In the distorted frequency domain, it can be expressed as

$$
\tilde{C}(j \Omega)=\frac{C_{1}}{c_{1}}+j \cdot \frac{1}{c_{1}}\left(C_{2} \Omega-\frac{1}{\Omega}\right) .
$$

These algorithms (12) and (15) are referred to as quasi-PID control in this paper.

With respect to higher-order and time-delay plants, the model system $K P_{m}(z)$ can be improved by

$$
K \tilde{P}_{m}(\delta)=\frac{K}{1+C_{1} \delta+C_{2} \delta^{2}} \cdot \mathrm{e}^{-L_{m} s},
$$


where $L_{m}$ is the inserted time-delay of the model system. Since the feedback compensator that has a time-lead characteristic cannot be realized, the quasi-PID controller should be written as:

$$
\tilde{C}(\delta)=\frac{1+C_{1} \delta+C_{2} \delta^{2}}{K\left(1+c_{1} \delta+c_{2} \delta^{2}\right)-z^{-d_{m}}}, \quad d_{m}=L_{m} / h .
$$

\section{SUM OF TRAPEZOIDAL AREAS}

The discretized nonlinear characteristics as shown in Fig. 2 is partitioned as follows:

$$
\begin{aligned}
& v^{\dagger}=\mathcal{N}_{d}\left(e^{\dagger}\right)=K e^{\dagger}+g\left(e^{\dagger}\right), \quad 0<K<\infty, \\
& \left|w^{\dagger}\right|=\left|g\left(e^{\dagger}\right)\right| \leq \bar{g}<\infty, \text { for }\left|e^{\dagger}\right|<\varepsilon, \\
& \left|w^{\dagger}\right|=\left|g\left(e^{\dagger}\right)\right| \leq \beta\left|e^{\dagger}\right|, \text { for }\left|e^{\dagger}\right| \geq \varepsilon
\end{aligned}
$$

in regard to $e^{\dagger} \in e$. Therefore, when analyzing the robust stability in a global sense, it is sufficient to consider the nonlinear term (21), because the nonlinear term (20) can be treated as a disturbance signal. In this study, since the nonlinear characteristic (19) is assumed to exist in the first and the third quadrant, the sector parameter $\beta$ should be considered in the following range:

$$
0 \leq \beta \leq K
$$

The robust stability of discretized systems as shown in Fig. 4 is analyzed based on the inner-product and norm analysis in the $\ell_{2}$ space. In regard to (21), the following new nonlinear function can be defined:

$$
f(e):=g(e)+\beta \cdot e .
$$

When considering the discretized output of the nonlinear characteristic, $w^{\dagger}=g\left(e^{\dagger}\right)$, the following expression is given:

$$
f\left(e^{\dagger}(k)\right)=w^{\dagger}(k)+\beta \cdot e^{\dagger}(k) .
$$

From inequality (21), it can be seen that the function (24) belongs to the first and third quadrants.

For the neutral points of $e^{\dagger}(k)$ and $w^{\dagger}(k)$, the following expression is given from (24):

$$
\frac{1}{2}\left(f\left(e^{\dagger}(k)\right)+f\left(e^{\dagger}(k-1)\right)\right)=w_{m}^{\dagger}(k)+\beta e_{m}^{\dagger}(k),
$$

where

$w_{m}^{\dagger}(k)=\frac{w^{\dagger}(k)+w^{\dagger}(k-1)}{2}, e_{m}^{\dagger}(k)=\frac{e^{\dagger}(k)+e^{\dagger}(k-1)}{2}$, The trapezoidal area of one-step transition on the grid pattern $f(e)$ is, therefore, written as follows:

$$
\begin{aligned}
\tau(k) & :=\frac{1}{2}\left(f\left(e^{\dagger}(k)\right)+f\left(e^{\dagger}(k-1)\right)\right) \Delta e^{\dagger}(k) \\
& =\left(w_{m}^{\dagger}(k)+\beta e_{m}^{\dagger}(k)\right) \Delta e^{\dagger}(k) .
\end{aligned}
$$

Here, $\Delta e^{\dagger}(k)$ is the backward difference of sequence $e^{\dagger}(k)$.

$$
\Delta e^{\dagger}(k)=e^{\dagger}(k)-e^{\dagger}(k-1) .
$$

Since $f\left(e^{\dagger}(k)\right)$ belongs to the first and third quadrants, the area of each trapezoid $\tau(k)$ is non-negative when $e(k)$ increases (decreases) in the first (third) quadrant. On the other hand, the trapezoidal area $\tau(k)$ is non-positive when $e(k)$ decreases (increases) in the first (third) quadrant.

In our study, the following assumption is provided with respect to the discretized response on a grid pattern.

Assumption. The absolute value of the backward difference of sequence $e(k)$ does not exceed $\gamma$, i.e.,

$$
|\Delta e(k)|=|e(k)-e(k-1)| \leq \gamma .
$$

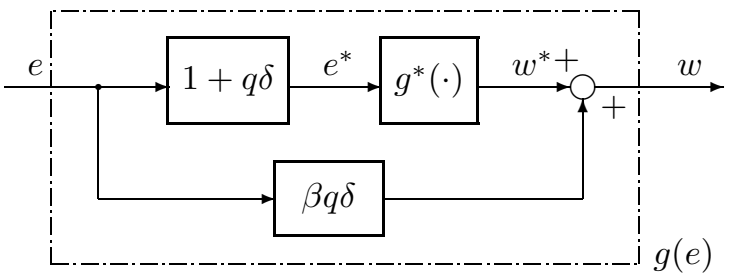

Fig. 5. Nonlinear subsystem.

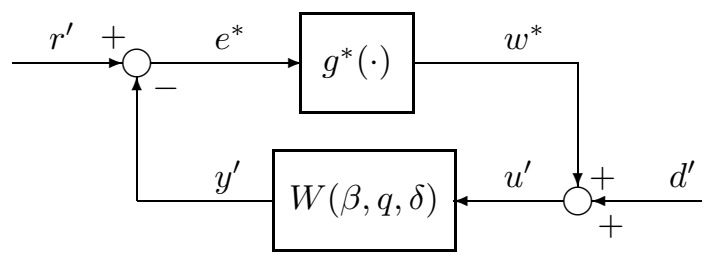

Fig. 6. Equivalent feedback system.

If condition (27) is satisfied, $\Delta e^{\dagger}(k)$ is exactly $\pm \gamma$ or 0 because of the discretization. That is, the absolute value of the backward difference can be given as

$$
\left|\Delta e^{\dagger}(k)\right|=\left|e^{\dagger}(k)-e^{\dagger}(k-1)\right|=\gamma \text { or } 0 .
$$

This assumption will be satisfied in the following examples.

Consider the following sum of trapezoidal areas:

$$
\sigma(p)=\sum_{k=1}^{p} \tau(k)
$$

If the above assumption is satisfied with respect to the discretization of the control system, the sum of trapezoidal areas, $\sigma(p)$, becomes non-negative for any $p$. Since the discretized output traces the same points on the stepwise nonlinear characteristic, the sum of trapezoidal areas is canceled when $e(k)$ (and $e^{\dagger}(k)$ decreases (increases) from a certain point $\left(e^{\dagger}(k), f\left(e^{\dagger}(k)\right)\right)$ in the first (third) quadrant. (Here, without loss of generality, the response of discretized point $\left(e^{\dagger}(k), f\left(e^{\dagger}(k)\right)\right)$ is assumed to commence at the origin.)

From equation (26), the sum of trapezoidal areas can be expressed as follows:

$$
\begin{aligned}
\sigma(p) & :=\frac{1}{2} \sum_{k=1}^{p}\left(f\left(e^{\dagger}(k)\right)+f\left(e^{\dagger}(k-1)\right)\right) \Delta e^{\dagger}(k) \\
& =\left\langle w_{m}^{\dagger}(k)+\beta e_{m}^{\dagger}(k), \Delta e^{\dagger}(k)\right\rangle_{p}
\end{aligned}
$$

Here, $\langle\cdot, \cdot\rangle_{p}$ denotes the inner product in the $\ell_{2}$ space,

$$
\left\langle x_{1}(k), x_{2}(k)\right\rangle_{p}=\sum_{k=1}^{p} x_{1}(k) x_{2}(k) \text {. }
$$

\section{ROBUST STABILITY IN A GLOBAL SENSE}

In order to derive the robust condition, the following new sequences are considered here:

$$
\begin{aligned}
& e_{m}^{* \dagger}(k)=e_{m}^{\dagger}(k)+q \cdot \frac{\Delta e^{\dagger}(k)}{h}, \\
& w_{m}^{* \dagger}(k)=w_{m}^{\dagger}(k)-\beta q \cdot \frac{\Delta e^{\dagger}(k)}{h} .
\end{aligned}
$$

where $q$ is a non-negative number. The relationship between equations (30) and (31) is as shown in Fig. 5. Based on these 
sequences, the following lemma is given (Okuyama et al. [1999]):

Lemma 1. If the following inequality is satisfied with respect to the inner product of the neutral points of (24) and the backward difference:

$$
\left\langle w_{m}^{\dagger}(k)+\beta e_{m}^{\dagger}(k), \Delta e^{\dagger}(k)\right\rangle_{p} \geq 0,
$$

the following inequality can be obtained:

$$
\left\|w_{m}^{* \dagger}(k)\right\|_{2, p} \leq \beta\left\|e_{m}^{* \dagger}(k)\right\|_{2, p} \leq \beta\left\|e_{m}^{*}(k)\right\|_{2, p}
$$

for any $q \geq 0$. Here, $\|\cdot\|_{2, p}$ denotes the Euclidean norm, which can be defined by

$$
\|x(k)\|_{2, p}:=\left(\sum_{k=1}^{p} x^{2}(k)\right)^{1 / 2} .
$$

Proof. The proof is given in (e.g., Okuyama [2006]).

By applying a small gain theorem to the loop transfer characteristic, the following robust stability condition for discretized nonlinear control systems can be derived (Okuyama [2008]).

Theorem 2. If there exists a $q \geq 0$ in which the sector parameter $\beta$ with respect to nonlinear term $g(\cdot)$ satisfies the following inequality, the discrete-time control system with sector nonlinearity (21) is robust stable in an $\ell_{2}$ sense:

$$
\beta<\beta_{0}=K \cdot \eta\left(q_{0}, \omega_{0}\right)=\max _{q \geq 0} \min _{\omega_{c} \geq \omega>0} K \cdot \eta(q, \omega),
$$

when the linearized system with nominal gain $K$ is stable. That is, the allowable sector can be given as $\left[0, \beta_{0}\right]$ from (34). Here, $\omega_{c}$ is a cut-off frequency that is defined based on the sampling theorem. The $\eta$-function is written as follows:

$$
\begin{aligned}
& \eta(q, \omega):= \\
& \frac{-q \Omega V+\sqrt{q^{2} \Omega^{2} V^{2}+\left(U^{2}+V^{2}\right)\left\{(1+U)^{2}+V^{2}\right\}}}{U^{2}+V^{2}}
\end{aligned}
$$

where $U(\omega)$ and $V(\omega)$ are the real and the imaginary parts of the loop transfer function $K P\left(\mathrm{e}^{j \omega h}\right) C\left(\mathrm{e}^{j \omega h}\right)$.

Proof. The loop transfer function from $w^{*}$ to $e^{*}$ can be given by $W(\beta, q, \delta)$ as shown in Fig. 6 , where

$$
W(\beta, q, \delta)=\frac{(1+q \delta) P(\delta) C(\delta)}{1+(K+\beta q \delta) P(\delta) C(\delta)},
$$

and $r^{\prime}, d^{\prime}$ are transformed exogenous inputs. Here, the variables such as $e^{*}, w^{*}, u^{\prime}$ and $y^{\prime}$ written in Fig. 6 indicate the $\delta$ transformed ones.

Based on the loop characteristic in Fig. 6, the following inequality can be given with respect to $\delta=j \Omega$ :

$$
\begin{gathered}
\left\|e_{m}^{*}(\delta)\right\|_{2, p} \leq k_{1}\left\|r_{m}^{\prime}(\delta)\right\|_{2, p}+k_{2}\left\|d_{m}^{\prime}(\delta)\right\|_{2, p} \\
+\sup _{\delta=j \Omega}|W(\beta, q, \delta)| \cdot\left\|w_{m}^{* \dagger}(\delta)\right\|_{2, p} .
\end{gathered}
$$

Here, $r_{m}^{\prime}(\delta)$ and $d_{m}^{\prime}(\delta)$ denote the $\delta$-transformation for the neutral points of sequences $r^{\prime}(k)$ and $d^{\prime}(k)$, respectively. Moreover, $k_{1}$ and $k_{2}$ are positive constants.

By applying the result of Lemma 1, the following expression can be obtained:

$$
\begin{gathered}
\left(1-\beta \cdot \sup _{\delta=j \Omega}|W(\beta, q, \delta)|\right)\left\|e_{m}^{*}(\delta)\right\|_{2, p} \\
\leq k_{1}\left\|r_{m}^{\prime}(\delta)\right\|_{2, p}+k_{2}\left\|d_{m}^{\prime}(\delta)\right\|_{2, p} .
\end{gathered}
$$

Therefore, if the following inequality is valid,

$$
\begin{aligned}
& |W(\beta, q, j \Omega)|= \\
& \left|\frac{(1+j q \Omega(\omega))(U(\omega)+j V(\omega))}{K+(K+j \beta q \Omega(\omega))(U(\omega)+j V(\omega))}\right|<\frac{1}{\beta} .
\end{aligned}
$$

the sequences $e_{m}^{*}(k), e_{m}(k), e(k)$ and $y(k)$ are restricted in finite values, when exogenous inputs $r(k), d(k)$ are finite and $p \rightarrow \infty$. From the square of both sides of inequality (39), the result of theorem is given.

\section{NYQUIST CURVE AND OFF-AXIS M-CIRCLES}

The design method is based on a Nyquist curve and off-axis M-circles. In the previous papers (Okuyama et al. [2002a,b]), the inverse function was used instead of the $\eta$-function, i.e., $\xi(q, \omega)=\frac{1}{\eta(q, \omega)}$. Using this notation, inequality (34) can be rewritten as follows:

$$
M_{0}=\xi\left(q_{0}, \omega_{0}\right)=\min _{q} \max _{\omega} \xi(q, \omega)<\frac{K}{\beta} .
$$

When $q=0$, the $\xi$-function can be expressed as:

$$
\xi(0, \omega)=\frac{\sqrt{U^{2}+V^{2}}}{\sqrt{(1+U)^{2}+V^{2}}}=\left|S_{c}\left(\mathrm{e}^{j \omega h}\right)\right|,
$$

where $S_{c}(z)$ is the complementary sensitivity function for the discrete-time system.

It is evident that the following curve on the complex plane,

$$
\xi(0, \omega)=M, \quad(M \text { : const. })
$$

corresponds to an $M$-circle in the Hall diagram. In this study, since an arbitrary non-negative number $q$ is considered, the $\xi$ function that corresponds to (41) and (42) is given as follows:

$$
\frac{U^{2}+V^{2}}{-q \Omega V+\sqrt{q^{2} \Omega^{2} V^{2}+\left(U^{2}+V^{2}\right)\left\{(1+U)^{2}+V^{2}\right\}}}=M \text {. }
$$

From this expression, the following quadratic equation can be obtained:

$\left(M^{2}-1\right) U^{2}+2 M^{2} U+\left(M^{2}-1\right) V^{2}+M^{2}-2 M q \Omega V=0$.

When $M>1$, the following is obtained from (44).

$$
\begin{gathered}
\left(U+\frac{M^{2}}{M^{2}-1}\right)^{2}+(V-\lambda)^{2}=\frac{M^{2}}{\left(M^{2}-1\right)^{2}}+\lambda^{2}, \\
\lambda=q \Omega M /\left(M^{2}-1\right) \geq 0 .
\end{gathered}
$$

Although the distorted frequency $\Omega$ is a function of $\omega$, the term $q \Omega=c_{q} \geq 0$ is assumed to be a constant parameter in this paper. Thus, it can been seen that (45) represents off-axis circles with their center at $\left(-M^{2} /\left(M^{2}-1\right), \lambda\right)$ and with radius of $\sqrt{M^{2} /\left(M^{2}-1\right)+\lambda^{2}}$.

Figure 7 shows an example of the modified Hall diagram and Nyquist curves of a control system with time delay. Here, $\mathrm{N}_{1}$ is a vector locus that contacts with an $M$-circle at the peak value $\left(M_{p}=\xi\left(0, \omega_{p}\right)=1.4\right)$. On the other hand, $\mathrm{N}_{2}$ is a vector locus that contacts with a circle $\mathbf{C}$ on the real axis, where all the $M$ circles cross the real axis. The latter case corresponds to the discrete-time system in which Aizerman's conjecture is valid (Okuyama et al. [1998]). At the continuous saddle point where is also the phase-crossover point $\mathrm{P}_{c}$, the following equation is satisfied:

$$
\left(\frac{\partial \eta(q, \omega)}{\partial q}\right)_{q=q_{0}, \omega=\omega_{0}}=0 .
$$

Evidently, the phase margin $p_{M}$ is obtained from the gaincrossover point $\mathrm{G}_{c}$. 


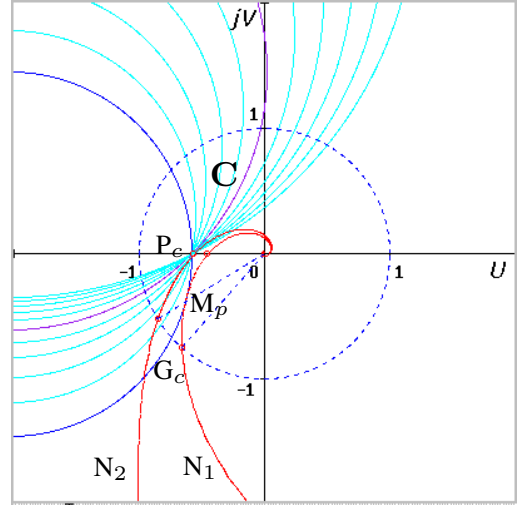

Fig. 7. An example of M-circles and vector loci.

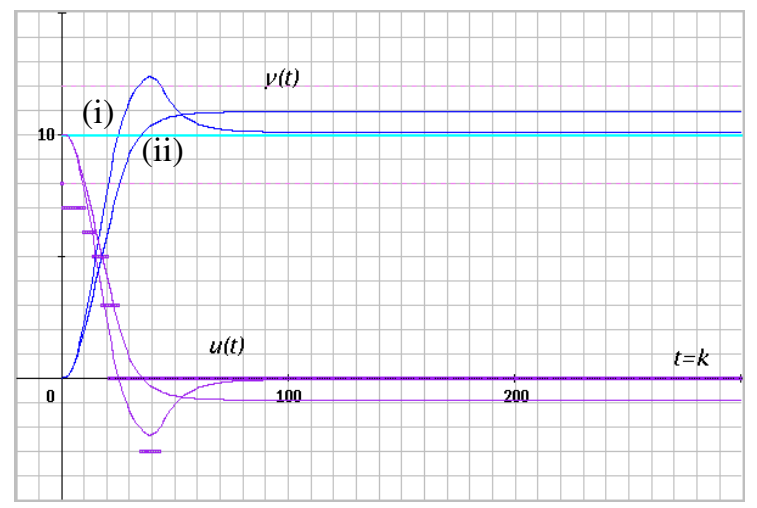

Fig. 8. Step responses for Example 1.

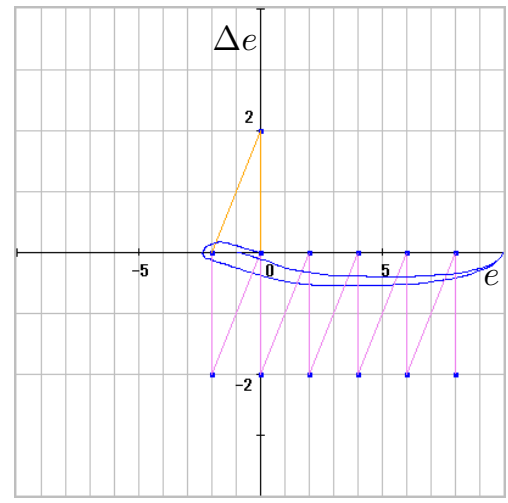

Fig. 9. Phase traces for Example 1.

\section{NUMERICAL EXAMPLES}

Example 1. Consider the following continuous plant:

$$
P(s)=\frac{K_{1}}{(s+0.1)(s+0.2)(s+0.5)},
$$

where the gain constant is $K_{1}=0.01$ and the sampling period is $h=1.0$. The discretized nonlinear characteristic considered here (discretized sigmoid, i.e. arc tangent) is as shown in Fig. 2. The resolutions of the input and output sequences of $\mathcal{N}_{d}(\cdot)$ are chosen as $\gamma_{1}=2.0$ and $\gamma_{2}=1.0$. When the nominal gain $K=1.0$ and the threshold $\varepsilon=2.0$ are considered, the sectorial area of the stepwise nonlinear characteristic for $\varepsilon \leq|e|$ can be determined as $[0.5,1.5]$. In this example, the resolutions of model system $K P_{m}(z)$ and feedback compensator $F(z)$ are defined as $\gamma_{c 1}=\gamma_{c 2}=\gamma_{2} / 25=0.04$.

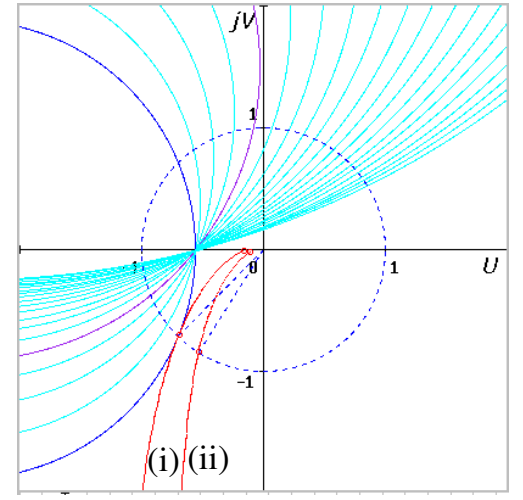

Fig. 10. M-circles and vector loci for Example 1.

As for the first case (i), the model system is chosen as follows:

$$
K \tilde{P}_{m}(\delta)=\frac{1}{1+8.0 \delta+8.0 \delta^{2}} .
$$

And the feedback compensator is defined as:

$$
\tilde{F}(\delta)=\frac{1+8.0 \delta+8.0 \delta^{2}}{1+8.0 \delta+\delta^{2}}
$$

The step response of (i) is depicted in Fig. 8. and the phase trace is as shown in Fig. 9. On the other hand, as for the second case (ii), the model system is chosen as:

$$
K \tilde{P}_{m}(\delta)=\frac{1}{1+8.0 \delta+8.0 \delta^{2}} z^{-4} .
$$

Since the sampling period $h=1.0$, the above (50) corresponds to the model system with time-delay $L_{m}=4.0$. The feedback compensator is the same as (49). In this case, the step response of (ii) is well stabilized and designed as shown in Fig. 8.

When the model and compensator are considered in high resolution, the equivalent controller can be written as

$$
\tilde{C}(\delta)=\frac{1+8.0 \delta+8.0 \delta^{2}}{8.0 \delta+\delta^{2}} .
$$

Therefore, the Nyquist plots and the off-axis M-circles of these cases is as shown in Fig. 10. (Hereafter, the cut-off frequency is chosen as $\omega_{c}=0.3$.) The parameter tuning and designing of the above model-reference control systems can be performed in this diagram. In either case, since the sector parameter $\beta$ becomes not less than $K=1.0$ from (22), it should be considered $\beta_{0}=1.0$. (i.e., the allowable sector is regarded as $[0.0,2.0]$.) Thus, the robust stabilty of the control system is guaranteed.

Example 2. Consider the following continuous plant with time delay:

$$
P(s)=\frac{K_{2}}{(s+0.1)(s+0.2)(s+0.5)} \mathrm{e}^{-L s},
$$

where $K_{2}=0.01$ and $L=4.0$. The resolutions of the model and compensator are the same as in Example 1. In addition, as for the first case (i), the model system and the feedback compensator are the same as (48) and (49), respectively. The step response of (i) is depicted in Fig. 11. On the other hand, as for the second case (ii), the model system and the feedback compensator are the same as (50) and (49). Also in this case, the step response is well stabilized as shown in Fig. 11. When the model system and the feedback compensator are in high resolution, the secor parameter becomes $\beta_{0}=0.78$ (i.e., the allowable sector is determined as $[0.22,1.78])$. Figure 12 shows the Nyquist plots and the off-axis M-circles of these cases. The parameter tuning and designing of the above model-reference 


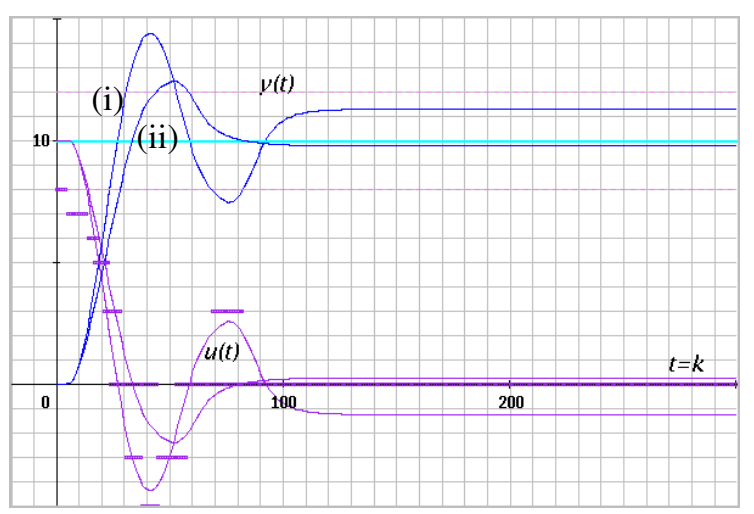

Fig. 11. Step responses for Example 2.

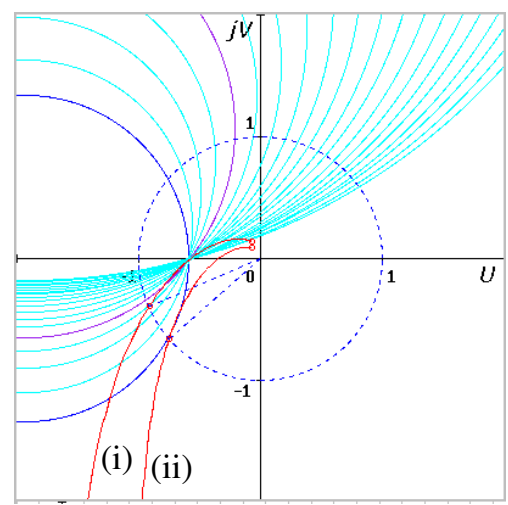

Fig. 12. M-circles and vector loci for Example 2.

control systems can be performed in this diagram. Thus, the robust stability of the control system is satisfied in either case of the above.

Example 3. Consider the following continuous plant with time delay:

$$
P(s)=\frac{K_{3}(s+0.3)(-s+0.5)}{(s+0.1)(s+0.2)(s+0.5)} \mathrm{e}^{-L s},
$$

where $K_{3}=0.05$ and $L=5.0$. This is an example of non-minimum phase plants. The resolutions of the model and compensator are the same as in Example 1. As for the first case (i), the model system and the feedback compensator are the same as (48) and (49). The step response of (i) is depicted in Fig. 13. On the other hand, for the second case (ii), the model system and the feedback compensator are the same as (50) and (49). (Here, the time delay of the model system is chosen as $L_{m}=0.5$.) Also in this case, the step response of (ii) is well stabilized as shown in Fig. 13. This is a counter example of Aizerman's conjecture.

\section{CONCLUSION}

This paper has described a designing problem of discretetime and discrete-value (discretized) control systems based on a model reference structure. The robust stability of that type of nonlinear control systems was examined in a frequency domain. The model reference feedback using a second-order continuous-value (linear) system was equivalently transformed into a traditional PID control. Based on the model reference structure, the robust stabilization and design of continuous plants could be achieved. The result of numerical examples show good performances and step responses. In general, the

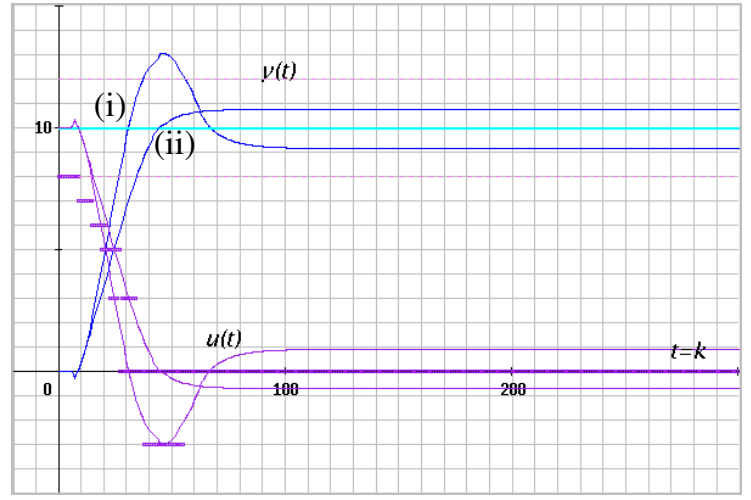

Fig. 13. Step responses for Example 3.

design method will be applied to digital and discrete control systems.

\section{REFERENCES}

D. F. Delchamps. Stabilizing a Linear System with Quantized State Feedback. IEEE Trans. on Automatic Control, volume 35, pages 916-924, 1990.

C. A. Desoer and M. Vidyasagar. Feedback System: InputOutput Properties, Academic Press, 1975.

N. Elia and S. K. Mitter. Stabilization of Linear System with Limited Information. IEEE Trans. on Automatic Control, volume 46, pages 1384-1400, 2001.

M. Fu. Robust Stabilization of Linear Uncertain Systems via Quantized Feedback. Proc. of the IEEE Int. Conf. on Decision and Control, TuA06-5, 2003.

R. E. Kalman. Nonlinear Aspects of Sampled-Data Control Systems. Proc. of the Symposium on Nonlinear Circuit Analysis, volume VI, pages 273-313, 1956.

Y. Okuyama et al.. Robust Stability Analysis for Nonlinear Sampled-Data Control Systems and the Aizerman Conjecture. Proc. of the IEEE Int. Conf. on Decision and Control, Tampa, USA, pages 849-852, 1998.

Y. Okuyama et al.. Robust Stability Evaluation for SampledData Control Systems with a Sector Nonlinearity in a GainPhase Plane. Int. J. of Robust and Nonlinear Control, volume 9, No. 1, pages 15-32, 1999.

Y. Okuyama et al. . Robust Stability Analysis for Non-Linear Sampled-Data Control Systems in a Frequency Domain. European Journal of Control, volume 8, No. 2, pages 99108, 2002.

Y. Okuyama et al. . Amplitude Dependent Analysis and Stabilization for Nonlinear Sampled-Data Control Systems. Proc. of the 15th IFAC World Congress, Barcelona, Spain, T-TuM08, 2002.

Y. Okuyama. Robust Stability Analysis for Discretized Nonlinear Control Systems in a Global Sense. Proc. of the 2006 American Control Conference, Minneapolis, USA, pages 2321-2326, 2006.

Y. Okuyama. Discretized PID Control and Robust Stabilization for Continuous Plants, Proc. of the 17th IFAC World Congress, Seoul, Korea, pages 1492-1498, 2008.

Y. Okuyama. Discretized PID Control and Robust Stabilization for Continuous Plants on an Integer-Grid Pattern Proc. of the European Control Conference Budapest, Hungary, pages 514-519, 2009.

F. Takemori and Y. Okuyama. Discrete-Time Model Reference Feedback and PID Control for Interval Plants. Digital Control 2000:Past, Present and Future of PID Control, Pergamon Press, pages 260-265, 2000. 